\title{
Strain gauge data logger for educational purposes
}

\author{
by SAMUEL K. CLARK, \\ Department of Applied Mechanics and Engineering Science, The University of Michigan, Ann Arbor, MI 48109
}

First presented at the B.S.S.M. 15th Annual Conference, Bristol, 3-6 Sept. 1979

Key words: Data logger, strain gauges, educational.

\section{Introduction}

For many years Strength of Materials laboratories in undergraduate engineering instruction have been taught using massive testing machines, and large specimens with their accompanying large loads. Such machines are obviously expensive and subject to damage. For this reason it has been traditional to conduct such experiments for undergraduate students on a demonstration basis, where the instructor actually operates the equipment, and the students stand by observing and taking notes.

This type of system was prevalent up until a few years ago and probably had some justification when strain measuring equipment was in its infancy, and had to be carefully adjusted by hand by people with years of experience in order to obtain consistently reliable results.

Almost all industrial strain measurement is now carried out by the use of resistance strain gauges, and these have become inexpensive enough so that students may themselves attach their own strain gauges for educational purposes, thus gaining invaluable practical insight into the difficulties associated with such types of measurements. However, instrumentation for the reading of strain has historically been very expensive and not readily available for undergraduate, individual, educational purposes. Recently the cost of electronic components has been reduced significantly in the United States by volume production of items which in some cases can be adapted to strain indicating equipment. This opens up the possibility of relatively inexpensive strain measuring instruments being available for instructional and general laboratory use. A description of one such instrument is the subject of this present paper.

\section{Philosophy of instruction}

For the past ten years, we have been endeavouring at the University of Michigan to bring individual strain measuring instruction directly to the student simultaneously with lectures in Strength of Materials. The Strength of Materials course taught there normally encompasses such topics as definitions of stress and strain, simple stress states, two dimensional stress states including Mohr's circle, the concepts of torsion and shear stress, beam bending and beam deflection. These lectures are normally accompanied by laboratory work involving simple definitions such as Young's modulus, stress-strain curves, Poisson's ratio measurements, and some work on torsion and an elementary measurement of a stress concentration. As previously described, this type of material was historically presented on a demonstration basis, but in 1965 a series of small scale testing machines were constructed using a design developed there. These machines use a hand or screw loading frame and are capable of exerting either tension, compression or torsion on relatively small specimens. At the time of this original development, little or no commercial equipment was available of this type. Since then, however, a number of individual bench type testing machines have come onto the market. many of them more flexible and versatile than those presently used in our laboratory. However, a typical machine of the type used at the University of Michigan is shown in Fig 1, and, while somewhat antiquated, it is seen that they are still suitable for the purposes originally intended.

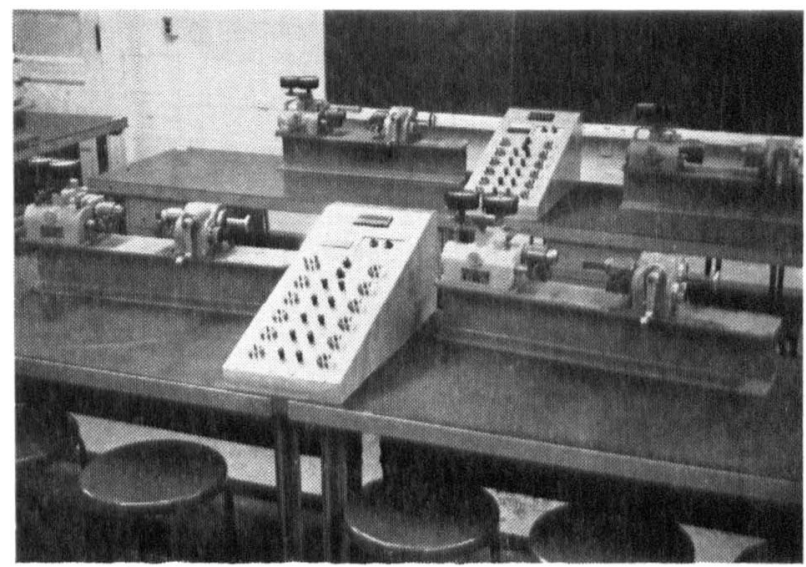

Fig 1. Loading machine and instrumentation station for materials testing instruction.

While these machines have been quite adequate for the educational purposes for which they were designed, our experience shows that the hydraulic type of load sensing elements seem to require substantially more maintenance than similar mechanical elements, and if the design were to be re-done today, a mechanical load indicating system would be recommended over a hydraulic one.

Under present crowded laboratory conditions two students must utilise a single testing machine at a time, which still gives considerable hands-on experience to each of the students involved. However, provisions for measuring strain are more cumbersome since it is necessary to utilise a single strain indicator connected through a multiple set of bridges with a switching system. This strain indicator is normally located between two testing machine stations, so that in actuality four students share the same strain indicator. These indicators are semi-mechanical in nature, having a mechanical servo controlled system with a zeroing circuit. This allows strain to be read directly on a counter wheel type of system. These also require a substantial amount of maintenance. More recently, digital indicating units of a relatively sophisticated type have been obtained for some of these stations, but their cost is still quite high. 
It appears that the logical course of development for bringing individual strain indicators into the hands of students would be a relatively inexpensive digital indicating strain meter capable of accepting either quarter, half or full bridges of either nominal 120 or $350 \mathrm{ohm}$ resistance. Such a system would have considerable teaching advantages, and, if the cost could be kept low, would be within the budget of many educational institutions.

\section{General layout of the strain indicator}

The strain indicator which is described in this paper was developed by Precision Measurement Co., Ann Arbor, MI, for the type of service just described in educational environments, but also serves as a research or industrial tool equally well. Its requirements are relatively simple. It must accept either quarter, half or full bridges in either 120 or $350 \mathrm{ohm}$ resistances, but need do so only through a very limited range of gauge factors, since experience shows that almost all gauges manufactured in the United States today carry gauge factors between 1.98 and 2.05 . For this restricted range of gauge factors, some circuit simplifications can be made, thus keeping the cost of the unit low.

An additional requirement on the unit is that it should have adequate balance control, so that bridge balance can be obtained with a wide range of gauge mismatches. This is accomplished using a typical balance circuit in the system.

A third requirement on the indicator is that it be capable of reading strains of the order of 10,000 to 15,000 microstrain. In order to achieve this with the type of circuit available to us, it was necessary to include in the system a multiplying circuit. The value of the multiplier chosen here was five-to-one, so that in cases of fairly large strains the multiplying switch for 5 can be activated, and the unit will indicate one-fifth of the actual strain.

While a number of excellent meter units has been available in the past, the delicacy and fragility of high quality meters seem to argue for a digital indicating unit for student use. If it were possible to develop such a unit, then its maintenance and life should be much greater than comparable meter devices. For that reason a fourth requirement on the unit was that it be a digital indicating system. While this may appear on the surface to be quite expensive, in fact the cost of light emitting diodes in the United States has been reduced considerably over the last few years, since they are widely used in a number of instruments and industrial applications. For that reason no significant cost penalty is associated with the use of light emitting diodes by themselves, although the auxiliary circuitry to drive them is substantially more complicated than would be encountered in the direct meter reading device.

The external appearance of the system involves the use of a number of input binding posts on the front face of the unit, which in proper combination are designed to accept either the quarter, half or full bridge configurations

A question concerns the type of power input for the system. Ideally for student use the unit should be powered from mains, which in the United States are 110 to 120 volts at $60 \mathrm{~Hz}$. However, the particular type of electrnoic circuit utilised here was borrowed from other industrial applications, and was designed to operate on 9 volts D.C.

Accordingly, it was felt that with very little additional cost, the unit could be made useful both for field and laboratory work by retaining the original battery excitation system and simply adding a regulated power supply which could be used alternatively for indoor use or for use where mains current is commonly available. For that reason an alternate power supply is available with the unit, and units so constructed can be used either in the field under battery power or on mains power when available.

An additional requirement on the unit is that it should have available oscillographic output in cases where dynamic strain measurements are to be recorded. While the system frequency response is not particularly high, such voltage output is extremely convenient for many purposes. It was found that by slight modifications of the original circuit, such an output could be added with relatively low cost, and it is included on the back panel of the instrument shown in Fig 2.

With such relatively simple controls, the user is immediately able to master the use of this strain indicator and can readily set up his own strain gauge bridge circuits for the measurement of strains during a two hour laboratory session. We feel that this is a considerable advantage over previous strain measuring instruments, particularly since the ruggedness of the device, without the use of a sensitive meter, makes it relatively free of maintenance.

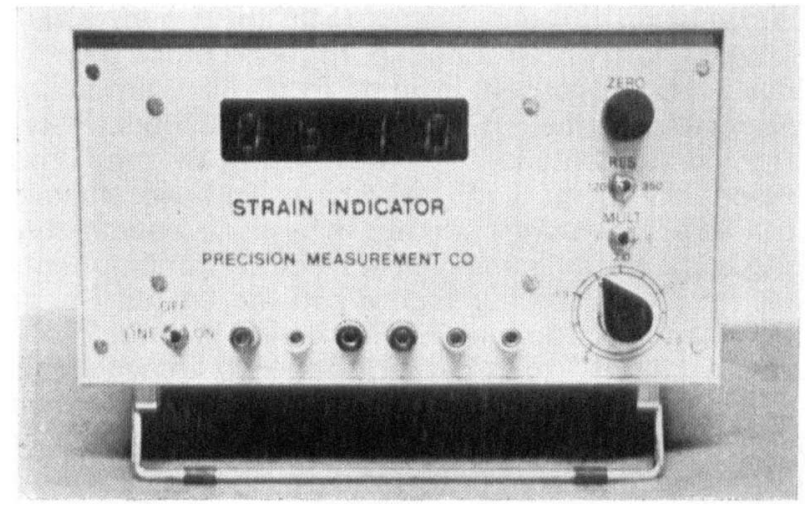

Fig 2. Front view of newly developed digital strain indiçator

\section{Circuit description}

A brief description of the general type of circuit used will be given here. In all cases, either quarter, half or full bridge operation is possible, using an internal set of additional resistors providing a full bridge array for the circuit. As strain causes resistance change in one or more of the arms of the bridge, an unbalanced voltage is developed and is fed to an amplifier. This amplifier is used to amplify the voltage output and filter extraneous noise, thus conditioning it for the analogue to digital converter portion of the circuit.

The analogue to digital converter consists of four basic stages, the integrator, comparator, oscillator and counters. Other circuits contribute to these stages and will be described briefly. The analogue to digital converter applies the dual slope (ramp down and ramp up) technique to convert analogue or input voltage into digital form. 
The integrator circuit has two inputs which control the state of its output. One input comes from the amplifier output voltage from the unbalanced bridge, while the other input remains fixed. The fixed input provides a reference level for switching the output to either high or low state. A time constant is provided which causes the output to ramp-down or ramp-up. When the input voltage is higher than the reference voltage, the output ramps down. When the input voltage is less than the reference voltage the output ramps up.

The comparator operates in a fashion similar to the integrator. It has two inputs, one fixed at the reference level which controls the state of its output. However, its output does not ramp-up or down. It is either at a high or low state since no time constant is involved.

The oscillator provides a standard of measurement for the counters, one oscillator being free running, which gives it a continuous series of pulses to the counters.

Four decade counters are used in the system, the first receiving pulses as its inputs from the free running oscillator. When the first counter counts nine pulses and starts counting the tenth, a pulse at its output is transferred to the input of the second decade counter. When the second counter reaches a nine to zero transition, a pulse is transferred from its output to the input of the third counter and so on. The total of four output counters then provides a four-digit readout system for the unit.

In addition to the basic operation of the system, which is widely used industrially for other purposes, a low battery indicator is present so that deficient batteries can be recognized by the appearance of a red dot on one of the light emitting diode units.

\section{Economic advantages}

Since one of the basic requirements of the design of this unit was to keep the cost low, a search was made for components which were in wide industrial application. The circuitry used in this system is almost all in large volume production for other industrial applications such as weighing systems, and for this reason the basic electronics are relatively inexpensive. The addition of the line voltage power supply and the analogue voltage output to an oscilloscope are units of relatively minor cost, which are added for the overall versatility and convenience of this strain indicator. However, the basic unit could be operated without these, although cost savings would be minimal. The majority of the cost in the unit is associated with the labour of constructing the circuitry and installing it properly in the case, along with the cost of the switches and potentiometers needed for the multiplying, balance and the gauge factor circuitry.

In spite of the versatility and usefulness of this unit, the resulting price has been kept sufficiently low so that there is wide expectation that it, or similar circuits, will rapidly come into the picture as teaching tools in engineering colleges, just as the small hand calculators have completely supplanted slide rules. They will also find wide application as general laboratory strain indicators, where they will release more expensive equipment for specialised testing.

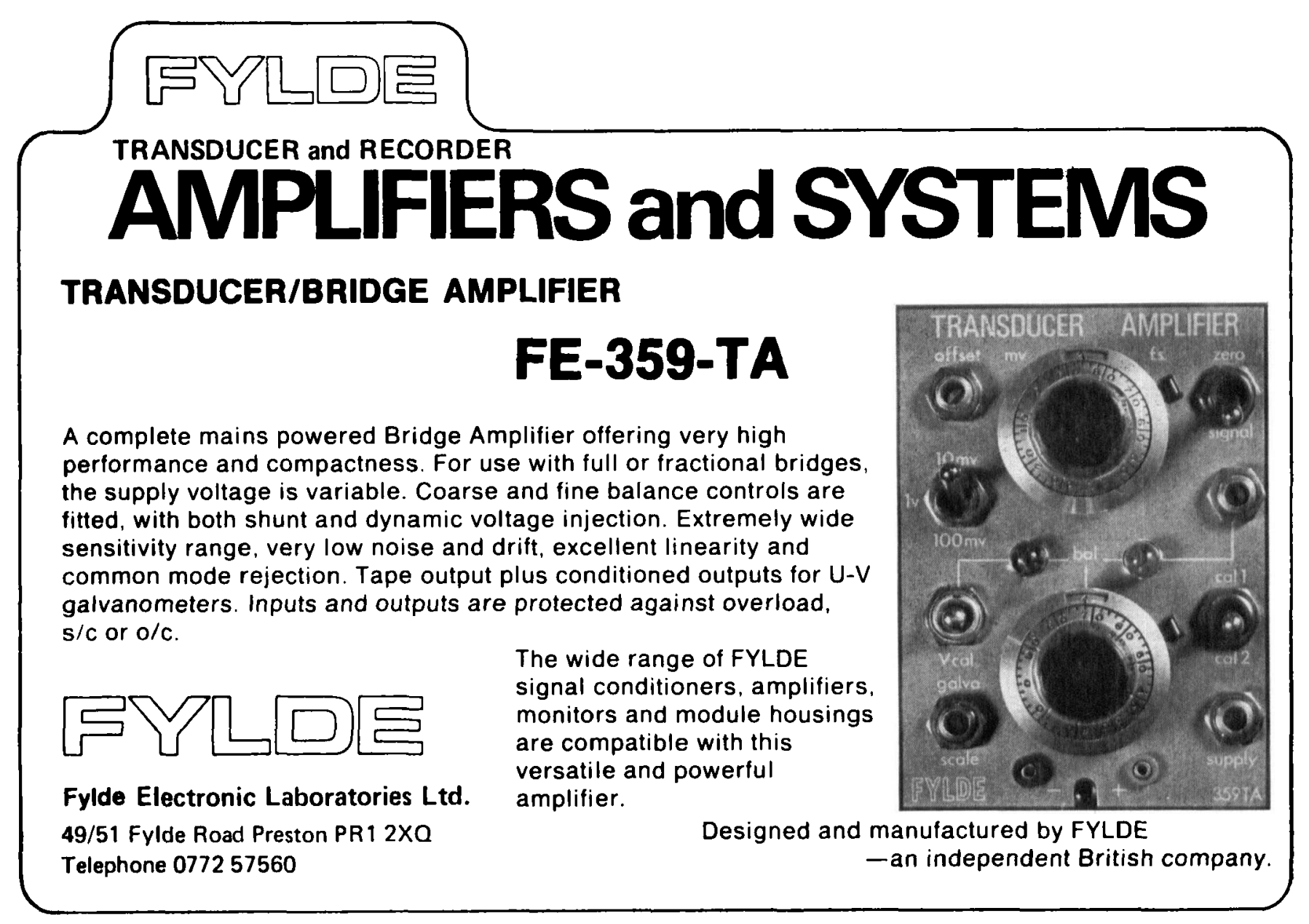




\section{B.S.S.M. CERTIFICATE SCHEME}

\section{DETAILS OF EXEMPTING COURSES FOR 1979-80 SEASON}

The following courses which provide exemption from Parts I and II of the scheme will be available at the institutions listed below.

\section{CITY OF BIRMINGHAM POLYTECHNIC:}

\section{Practical Strain Gauging (Part I exemption)}

5 days: 19-23 May 1980

Designed for technician engineers whose responsibility it is to carry out strain gauge installation. High and low temperature bonding techniques, associated instrumentation and data recording procedures are dealt with. During the course all members will design, produce and test their own precision strain-gauged transducer. A thoroughly practical course.

\section{Advanced Strain Measurement (Part II exemption)}

\section{5 days: $16-20$ June 1980}

This course is essentially a collection of twenty lectures on specialist topics, the lecturers, all of whom are recognised experts in their respective fields, being drawn from industry and universities. Unlike the other Strain Measurement courses which are of an introductory nature and highly practical in content, this course seeks to provide advanced knowledge of the more specialised techniques required to cope with particularly difficult problems such as elevated temperature measurements, measurement on non-metallic materials, etc. It also seeks to provide an introduction to the more recently introduced techniques such as Moiré, and Holography and should appeal to all personnel engaged in strain measurement/stress analysis activities at any level.

For full details of all courses contact:

Dr E. J. Hearn, Department of Mechanical and Production Engineering, City of Birmingham Polytechnic, North Centre, Franchise Street, Perry Barr, Birmingham, B42 2 SU.

\section{NORTH EAST LONDON POLYTECHNIC}

\section{Basic Photoelestic Techniques (Part I exemption)}

Five consecutive Tuesdays 09.00-17.00 8 January-12 February 1980

Introduction to experimental stress analysis techniques; Basic Photoelastic principles and applications; Two-dimensional transmission techniques including calibration, isoclinics and stress trajectories, boundary stress determination, compensation procedures, and simple stress duction to three-dimensional techniques: Photoelastic coating techniques: Contouring and cementing of coatings including practice; Errors in measurements; Project involving detailed analysis of an engineering component using coating or transmission technique.

Strain Gauges and Brittle Lacquer (Part I exemption) Five consecutive Tuesdays 09.00-17.00 6 May-10 June 1980

Introduction to experimental stress analysis techniques; Basic strain gauge principles; Strain gauge instrumentation ; gauge factor ; bridge configurations, compensation techniques, a.c.-d.c. systems; Selection of gauges, leadwires, adhesives and protective coatings : Practical demonstrations and practice in the bonding of strain gauges using various types of adhesive; Measurement of torque, bending, end load, pressure, etc.; Use of strain gauge rosettes and strain circle constructions; Recording techniques including data logging; Brittle lacquers-principles and practice; Transducer design and calibration project; Dynamic measurements: Errors in measurements.

\section{PART III ASSESSMENT}

The third set of Part III B.S.S.M. Certificate Examinations will be held during the week commencing 23rd June 1980. Persons who have passed Parts I and II of the Certificate Scheme or who have gained exemption from these parts are requested to apply for registration to the Examination on Form C3 before 18t Mey 1980. These forms are available from the B.S.S.M. Administration Officer, 281 Heaton Road, Newcastle upon Tyne NE6 50B.

For candidates who have only gained exemption from Part I (and others who consider they have a just claim for exemption) a special short course providing exemption from Part II will be held during week 16th-20th June 1980. Details of this course together with details of procedures for exemption claims may also be obtained from the B.S.S.M. Administration Officer.

\section{NOTICE TO UNIVERSITIES, POLYTECHNICS AND COLLEGES}

\section{Courses providing exemption from parts of the B.S.S.M. Certificate Scheme}

The pilot period for the B.S.S.M. Certificate scheme based on courses run exclusively at the City of Birmingham Polytechnic is now complete. Institutions seeking approval for courses to provide exemption from any part(s) of the scheme are invited to apply to the B.S.S.M. Administration Officer. Commercial organisations running training courses may also wish to apply. 